Voix et Images

volxetimages

\title{
Un martyrium remarquable, mais insolite
}

\section{Robert Major}

Volume 15, numéro 2 (44), hiver 1990

Pratiques illicites

URI : https://id.erudit.org/iderudit/200841ar

DOI : https://doi.org/10.7202/200841ar

Aller au sommaire du numéro

\section{Éditeur(s)}

Université du Québec à Montréal

\section{ISSN}

0318-9201 (imprimé)

1705-933X (numérique)

Découvrir la revue

\section{Citer cet article}

Major, R. (1990). Un martyrium remarquable, mais insolite. Voix et Images, 15(2), 278-282. https://doi.org/10.7202/200841ar d'utilisation que vous pouvez consulter en ligne.

https://apropos.erudit.org/fr/usagers/politique-dutilisation/ 


\section{Essai}

\section{Un martyrium remarquable, mais insolite}

\section{par Robert Major, Université d'Ottawa}

Sans doute est-ce justice que, dans un numéro de Voix \& images offrant un dossier sur les pratiques "illicites", on rende compte de l'œuvre de Guy Laflèche, les Saints Martyrs canadiens ${ }^{1}$. Il fut un temps, en effet, et pas si lointain, où les pratiques illicites - littéraires ou autres - étaient interdites, justement, prohibées et condamnées, ou du moins passées sous un silence réprobateur. Ce sont les saints martyrs, canadiens ou autres, qui retenaient l'attention, faisaient les manchettes ou la une des revues, ou motivaient les gens à descendre dans la rue pour des manifestations monstres qu'on appelait, à l'époque, processions, croisades ou congrès. Les rôles sont maintenant inversés. Lillicite affirme son intérêt, sinon sa légitimité, réclame sa place au soleil; et c'est le père Jogues et consorts, le père Jogues - qui, pourtant, obéissait aux plus petits dans les choses licites pour basses qu'elles fussent (II, 98) - qui, paradoxe, est devenu illicite, qu'on enferme à double tour dans le placard le plus négligé de notre mémoire collective. Autrefois on croyait que cétait crime de se taire devant une vie si exemplaire (Depuis ce temps jusqu'à sa mort, il s'est passé quantité de choses fort remarquables qu'on ne peut sans crime dérober au public, puisqu'elles sont honorables à Dieu et pleines de consolation pour les âmes qui aiment à souffrir pour Jésus-Christ, II, 41); maintenant on ne veut plus taire le crime qui trouve facilement ses champions sur la place publique et même ses apologistes et analystes dans de très sérieuses revues universitaires. $O$ tempora, o mores.

Guy Laflèche, cela se devine par ses livres récents et je le dis sans connaître la personne, me semble l'homme du contre-courant. Il aurait été du convoi qui remontait le Saint-Laurent et l'Outaouais vers la Huronie, luttant contre le cours des eaux, ahanant contre le fil, comme ces jésuites qu'il s'est donné pour mission de suivre à la trace dans les forêts du Nouveau-Monde. Serait-il revenu de Huronie? Cela aurait signifié se laisser porter par le flux. On peut en douter. L'homme du contre-courant, donc, et à un double titre. D'abord, parce qu'il s'intéresse passionnément à un sujet que, depuis plusieurs années déjà, les Québécois pensent avoir relégué aux oubliettes; ensuite parce qu'il s'est lancé dans une entreprise colossale par ses dimensions, vieille de plusieurs années déjà (son édition de la Relation de Paul Lejeune est de 1973) et qui exigera encore plusieurs années (le cinquième et dernier volume de cette œuvre est prévu pour 1998 !). Un quart de siècle, et davantage, pour se donner le plaisir de tout savoir sur les Saints Martyrs canadiens (I, 9). Dans un milieu littéraire où chacun s'active à courir de colloque en congrès, avide de sauter dans le dernier train en marche, et où une publication est le plus souvent un recueil de papiers collés, 
cette singulière détermination et cet engagement tenace, cette volonté de mener à terme un vaste projet personnel, loin de la presse et du bruit, me semblent exemplaires, remarquables, dignes d'être chaleureusement accueillis.

Coup sur coup, Guy Laflèche a publié les deux premiers volumes de son œuvre quinée. Le premier présentait, dans sa partie la plus volumineuse, une bibliographie analytique et critique du sujet: l'auteur posait donc au portail ce qui est habituellement refoulé à la fin d'une œuvre. Il étalait ainsi devant le lecteur, d'entrée de jeu, tous les documents qui traitent de près ou de loin des Martyrs canadiens, classait et jugeait ces écrits, avec une verve et une vigueur qui ne sont guère l'apanage des bibliographes. Il se livrait ensuite avec délices à un examen minutieux du parcours par lequel ces événements étaient devenus un mythe. Son propos se voulait essentiellement démystificateur: ces saints Martyrs canadiens ne sont ni des martyrs (ils n'ont pas été persécutés à cause de leurs convictions religieuses, ils ne sont pas morts plutôt que d'abjurer leur foi), ni des Canadiens. Et pourtant, on a fait de ces événements l'épisode central et séminal de l'épopée nationale.

Sans subterfuges donc, Guy Laflèche annonce ses couleurs dans le premier volume, Histoire du mythe ${ }^{2}$. Le deuxième volume, le Martyre d'Isaac Jogues par Jérôme Lalemant, présente l'édition critique de six chapitres de la Relation de 1647, rédigée par le supérieur des missions en Nouvelle-France à ce moment, Jérôme Lalemant: les six chapitres ayant trait au père Isaac Jogues.

Le livre est divisé en deux parties - le texte d'une part, les notes et commentaires critiques d'autre part - introduites par une brève présentation et complétées par une chronologie et un index. Les notes et commentaires constituent l'essentiel du volume - plus des deux-tiers, en petits caractères - mais tout cet appareillage critique ne se justifie que par l'intérêt intrinsèque du texte lui-même. Qu'en est-il donc de celui-ci?

Reconnaissons d'emblée que cette narration est du plus haut intérêt, et susceptible de capter l'attention d'une grande diversité de lecteurs. Sa portée historique est indéniable: il s'agit d'un document fondamental sur notre histoire, celle de la Nouvelle-France, certes, mais aussi celle de l'Église canadienne, de la sensibilité religieuse occidentale, des États-Unis d'Amérique et de la présence amérindienne. De plus, ce document est riche d'enseignements anthropologiques et ethnologiques, en particulier sur le conflit radical des civilisations, sur la pénible intégration des Européens en Amérique, et sur la non moins douloureuse assimilation de l'Europe par les Amérindiens. Intérêt multiple et foisonnant, donc. Mais c'est sans doute la qualité littéraire du texte qui doit davantage nous retenir.

Celui-ci se veut direct, sans apprêts et sans artifices: en somme, une simple relation. En réalité, il est d'une surprenante modernité et d'une savoureuse complexité. Guy Laflèche a beau jeu de montrer, par exemple, comment lintertexte fondamental de la Passion du Christ impose son schéma narratif à cette relation des tribulations du père Jogues: capture, violences, dénuement, bastonnades, marche forcée, chutes, crucifixion et mise à mort, sur fond de traîtrise et de sacrifice librement consenti, conformément aux volontés du Père (Père supérieur, dans ce cas), tout y est. Cette narration obeit aux exigences d'un Texte premier, dont elle ne fait qu'actualiser les virtualités. De plus, elle les actualise 
par des procédés d'une étonnante modernité. En effet, la relation de Jérôme Lalemant est en grande partie le collage, la mosaïque ou la refonte de textes antérieurs, de type biographique ou autobiographique: lettres ou mémoires de Jogues lui-même, rédaction de son supérieur immédiat et de son confesseur, correspondances diverses, dont certaines venant des Hollandais, témoignages de compagnons de capture, qu'ils soient Blancs, Hurons ou Algonquins. Tout cela est coulé dans le creuset de Jérôme Lalemant, qui se livre à une opération textuelle fascinante, tenant du bricolage, de la réécriture (avouée ou subreptice), de la citation (reconnue ou refoulée), de la glose, de la paraphrase, du soulignement et de linvention. Opération où constamment le sens glisse, dérape, prend des teintes nouvelles, s'enrichit de connotations insoupçonnées. Guy Laflèche, fin pisteur, excelle à démêler cet écheveau. Quand on sait, de plus, que cette opération est au service d'une narration souvent palpitante dont certains passages - je pense à la fuite de Jogues, par exemple - sont dignes des romans de Fenimore Cooper, on est comblé. Et on comprend que Guy Laflèche ait mis en exergue cette phrase percutante du cinquième chapitre:

La Reine ayant oui parler de ses souffrances dit tout haut, on feint des Romans, en voilà un véritable, entre-meslé de grandes aventures.

Exergue tout à fait adapté à la sensibilité littéraire actuelle et provocante pour les casuistes littéraires contemporains. Romans feints, roman véritable: merveilleuse dichotomie, où le vrai et le faux, l'authentique et le factice, le fabriqué et le vécu s'entrechoquent et laissent le lecteur songeur.

Cette narration est donc un document d'une qualité littéraire certaine, d'une réelle importance, et d'une lecture passionnante. Et d'autant plus que Guy Laflèche s'est donné un mal fou pour l'établir dans son authenticité et dans toute sa saveur d'origine. En effet, il avait à transcrire un texte qui, au départ, avait été édité rapidement et sans soins particuliers (il s'agit d'une publication populaire, largement diffusée, ne l'oublions pas, pleine de coquilles et de fautes) et, de plus, publié à un moment où l'usage orthographique n'était pas rigidement fixé. Il l'a fait avec une extrême rigueur, s'aidant de programmes informatisés divers pour atteindre une transcription d'une fidélité exemplaire, d'une grande lisibilité malgré l'orthographe du XVII ${ }^{e}$, rajeunie parce que la graphie est modernisée (miffion devient mission, par exemple), mais authentique parce que l'uniformisation s'est effectuée en conformité avec les règles mêmes du corpus, patiemment établies. Les pages que Guy Laflèche consacre à l'"Édition " (p. 103-126), tout en étant fort techniques, constituent un excellent survol des difficultés d'établissement d'un texte littéraire ancien et une introduction vivante à l'histoire de la langue et de l'édition.

Étant donné ce protocole d'édition, axé sur la régularisation et la normalisation, cette édition critique ne présente pas de variantes au sens strict. Dans le chapitre des "Variantes " (p. 127-150), sont plutôt mis en évidence: d'abord les Ti, les textes inclus dans le texte de Lalemant, c'est-à-dire qu'il utilise en les présentant comme tels; ensuite les Ts, les textes source qu'il utilise sans les citer mais quil pille à sa guise; finalement les $\mathrm{Tc}$, textes contemporains non utilisés par Lalemant mais dont la connaissance éclaire l'un ou l'autre aspect de sa 
relation. Ces " variantes " permettent de constater le bricolage auquel s'est livré le rédacteur. Toutefois, l'enseignement qu'il faut tirer de ces renseignements sur la matérialité du texte se trouve abondamment exploité dans la partie suivante, les "Notes", commentaire multiple sur le contenu du texte lui-même.

Ces notes (p. 151-283) sont d'une très grande richesse, d'une précision tatillonne et d'un intérêt soutenu. Elles peuvent aussi bien porter sur la qualité littéraire du texte ou les enjeux de cette narration que sur la situation politique ou sociologique; elles peuvent dresser avec minutie la biographie d'un personnage, protagoniste ou figurant, Blanc ou Amérindien, ou fixer la chronologie. À chacune de ses articulations, le texte est copieusement commenté et le recoupement des notes, le chassé-croisé de la glose tissent un réseau d'explications d'une cohérence remarquable. C'est indéniablement ici que Guy Laflèche excelle: la richesse de son fichier et la maitrise de sa matière donnent à ces notes une force et un pouvoir de conviction indéniables, même lorsqu'il échafaude des hypothèses. C'est le cas, par exemple, lorsqu'il s'aventure à expliquer, avec force détails et conjectures, des silences dans la narration. Pourquoi les Hurons captifs ne sont-ils pas identifiés (n. 36, chap. 2)? Sans doute parce que cela témoignerait des pauvres résultats de la mission huronne... Pourquoi Jogues ne mentionne-t-il jamais Oionhaton, Huronne de premier plan, éduquée chez les Ursulines, captive comme lui (ibid.)? Parce que celle-ci, tout en restant chrétienne, a été intégrée à l'Iroquoisie et deviendra puissante matronne, bien que chrétienne. Donc, ce n'est pas parce qu'il est chrétien que Jogues sera tué, mais bien pour d'autres raisons... Pourquoi n'a-t-on pas interrogé Guillaume Couture, autre compagnon de capture, au moment de rédiger l'hagiographie de Jogues (n. 17, chap. 5)? Sans doute parce qu'il s'est marié à une Iroquoise, ce qu'on passe évidemment sous silence... Ces hypothèses, et d'autres (la politique du tribut des Iroquois, par exemple, n. 33, chap. 6) sont patiemment étayées (cette dernière note compte six pages d'un texte serré...) et remporteront sans doute l'adhésion de la majorité des lecteurs informés.

Mais même le lecteur le plus sympathique à cette entreprise d'envergure ne pourra accepter le procès d'intention que Guy Laflèche intente à Jérôme Lalemant. Qu'on s'arrête à l'ambiguïté du sous-titre de ce deuxième volume: le Martyre d'Isaac Jogues par Jérôme Lalemant. Pour Guy Laflèche, Lalemant est l'auteur de ce martyre dans les deux sens du mot: le rédacteur du texte qu'on lit et la cause première de l'événement qui est narré dans le texte. Voici donc que l'expression " roman véritable " s'éclairerait d'un sens radicalement nouveau: il ne s'agit pas de simplement copier, refléter, reproduire la vérité: il faut d'abord créer l'événement qu'on se propose de narrer... Lalemant, écrivain génial, en somme, mais horrible, monstrueux, exécrable. C'est lui qui aurait sciemment envoyé Isaac Jogues à sa mort. Si Jogues n'avait pas été tué d'un coup de hache en octobre 1646, lors d'une mission diplomatique injustifiée, ses supplices de l'automne 1642 et de l'hiver 1643 auraient été sans utilité. Lalemant doit trouver le moyen de le faire mourir violemment, pour en faire un martyr, de peur quil ne décède d'une vulgaire pneumonie. Il gommera ensuite le plus possible l'hiatus entre les supplices sans mise à mort et la mort ultérieure sans supplices. 
Guy Laflèche ne lui pardonne ni la « décision* présumée de sacrifier Jogues ni sa façon triomphaliste et sans vergogne (p. 19) de rapporter ce sacrifice ${ }^{3}$. Il lui imagine un sourire béat au moment où il rédigeait sa relation, puis il décrète ce sourire insupportable! Il lui prête un visage sans douleur, ni regret, ni angoisse (II, 9), puis s'acharne sur ce froid technocrate (II, 155) qu'il a inventé de toutes pièces! Avouons que le procédé est très gênant. Et sans doute les partisans d'une histoire non passionnelle voudront-ils sauter à pieds joints par-dessus la «Présentation* du volume. Les autres, eh bien, ils y découvriront une autre facette du talent de Guy Laflèche, bien saisie par la Reine trois siècles avant sa naissance: le plaisir trouble que lui et d'autres peuvent prendre à feindre des romans.

1 Guy Lafleche, les Saints Martyrs canadiens, vol. I: Histoire du mythe, Laval, Editions du Singulier, 1988, 364 p. (avec 16 pages de planches hors-terte); vol. II: le Martyre d'Isaac Jogues par Jérôme Lalemant, Laval, Éditions du Singulier, 1989, 330 p.

2 Je me permets de renvoyer à mon compte rendu du premier volume dans Lettres québécoises, n० 54, été 1989 , p. 45-46.

3 Le lecteur, médusé par tant d'acharnement contre Lalemant, ne peut que se demander: mais quelle autre faģon un croyant, un missionnaire, un jésuite, un propagandiste religieux, en ce début du XVIIe siècle, convaincu que le sens même de la vie était de souffrir pour Jésus-Christ et que le sacrifice librement consenti était à la fois purificateur et salvateur, salvateur de soi et des autres et au premier titre de ses bourreaux, quelle autre façon, donc, un tel rédacteur aurait-il bien pu prendre? D'ailleurs, dire que Jogues voulait se dérober et qu'il fut un agneau sacrificiel récalcitrant, contre $8 a$ volonté la plus manifeste (II, 10), est une affirmation qui ne résiste pas à la comparaison avec ses propres affirmations épistolaires. Jirai mais je ne reviendrai pas, dit-il, bien conscient de ce qui l'attend, mais je serois heureux si Nostre Seigneur vouloit achever le sacrifice où il l'a commence (II, 90). 